\title{
Management of Inflammatory Cardiac Masses
}

\author{
Ankur A. Karnik ${ }^{\mathrm{a}, \mathrm{b}}$, Eric H. Awtry ${ }^{\mathrm{a}}$
}

\begin{abstract}
Systemic rheumatologic and inflammatory disorders can affect almost any organ system, including the heart. The cardiac valves, conduction system, myocardium, endocardium, pericardium, and coronary arteries may be affected. Intracardiac masses may develop as part of the disease process or a consequence of their therapy, such as methotrexate-associated nodulosis. Optimal therapy in these cases is not known, since many patients are asymptomatic and the potential benefit of surgical excision must be weighed against its associated morbidity and mortality. Importantly, these inflammatory masses must be differentiated from thrombus, infection, and primary and metastatic tumors. We present three cases of inflammatory cardiac masses associated with rheumatoid arthritis and Wegener's granulomatosis, which were successfully treated conservatively, and propose a management algorithm. The benefits of such an approach must be individualized and weighed against the risks of systemic embolization, stroke and obstruction.
\end{abstract}

Keywords: Cardiac mass; Inflammatory; Rheumatoid arthritis; Wegener's granulomatosis

\section{Introduction}

Primary cardiac tumors are extremely rare and are seen in $0.001 \%$ to $0.3 \%$ of individuals based on autopsy series; $75 \%$ are non-malignant [1]. Taken together, primary tumors and tumor-like conditions vary widely in appearance, location, size, number, and age at presentation [2]. Many of these tumors arise in the setting of genetic syndromes [2]. While recommendations for surgical excision depend on presumed etiology and presence of symptoms or obstructive physiology, some tumors such as hemangiomas [3] and inflammatory myofibroblastic tumors (IMT) [4-6] have been known to regress either spontaneously or after treatment with steroids. There are several prior reports of cardiac masses associated with systemic disorders such as Wegner's granulomatosis $[7,8]$, sarcoidosis [9,

Manuscript submitted October 26, 2018, accepted November 12, 2018

aSection of Cardiology, Department of Medicine, Boston Medical Center and Boston University School of Medicine, Boston, MA 02118, USA

${ }^{\text {b} C o r r e s p o n d i n g ~ A u t h o r: ~ A n k u r ~ A . ~ K a r n i k, ~ C o l l a m o r e ~ 8, ~ D i v i s i o n ~ o f ~ C a r d i o-~}$ vascular Medicine, Boston Medical Center, 88 East Newton Street, Boston, MA 02118, USA. Email: Ankur.karnik@bmc.org

doi: https://doi.org/10.14740/cr801
10], Behcet's disease [11, 12] and rheumatoid arthritis [13-15]; however, owing to the rarity of these masses, there is currently no consensus regarding management. We present three cases of patients with cardiac masses occurring in association with systemic inflammatory disease whose lesions significantly diminished with conservative therapy, review the English language literature regarding such an approach, and suggest an algorithm for management of these types of masses. Given a sample size of three, this case series was exempt from review by the Boston University Institutional Review Board.

\section{Case Reports}

\section{Case 1}

A 63-year-old woman with breast cancer status post lumpectomy and radiation, rheumatoid arthritis, and paroxysmal atrial fibrillation, presented with diplopia and was found to have multiple embolic strokes. She had multiple flares of her rheumatoid arthritis in the past, including an inflammatory pericardial effusion, but it was currently in remission on methotrexate and certolizumab. Brain magnetic resonance imaging (MRI) revealed thalamic and cerebellar infarcts. Transesophageal echocardiography (TEE) demonstrated sessile masses in the left atrium (LA) and right atrium (RA) that were contiguous with the interatrial septum, as well as a pedunculated mass in RA (Fig. 1a, b) (Supplementary Videos 1a, b) (www. cardiologyres.org). Cardiac MRI confirmed biatrial masses with a heterogenous enhancement pattern suggesting vascularization, most consistent with an inflammatory or neoplastic process and not a thrombus. Surgical resection was not felt to be feasible because of the multiple locations of these lesions, which suggested a systemic process. She was started on warfarin (goal INR 2 - 3) with a heparin bridge given a history of paroxysmal atrial fibrillation with multiple embolic strokes, treated with prednisone for rheumatoid arthritis-related tenosynovitis, and her methotrexate was stopped. Repeat TEE 2 months later revealed near-complete resolution of the atrial masses (Fig. 1c). Cardiac MRI 2 years later showed a mobile avascular echodensity in the RA most consistent with thrombus (different from prior study). She was lost to follow-up so it is unknown if she was still on warfarin at that time.

\section{Case 2}

A 75-year-old woman with rheumatoid arthritis and paroxysmal atrial fibrillation presented with dysarthria and inattention 

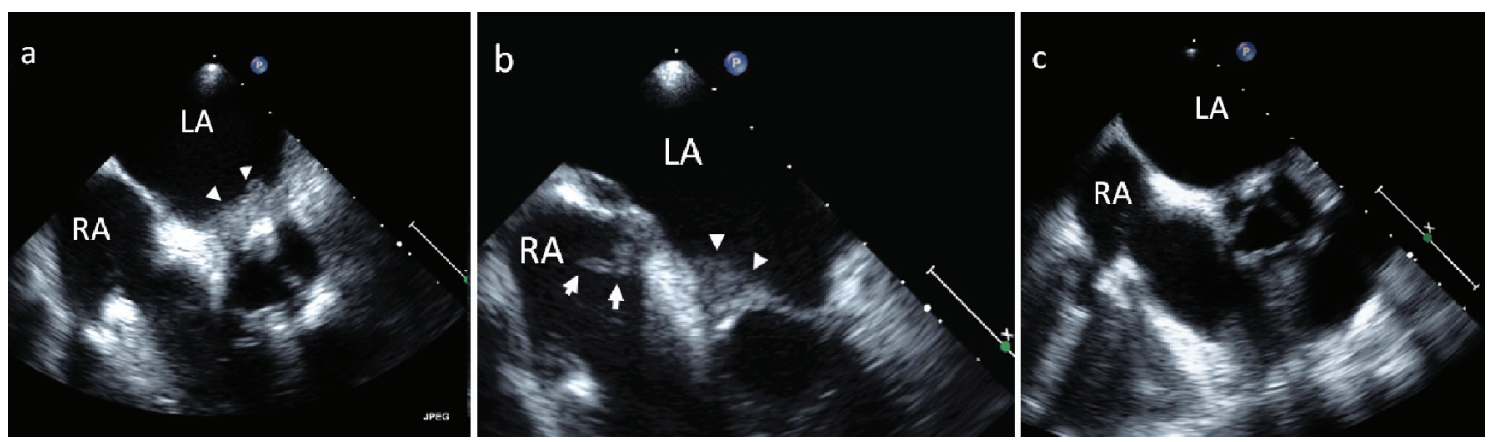

Figure 1. $(a, b)$ TEE of sessile masses in the LA and RA (arrow heads) as well as a pedunculated mass in the RA (arrow with tail) in a patient with rheumatoid arthritis. (c) TEE 2 months after initiation of warfarin, prednisone, and cessation of methotrexate shows near-complete resolution of the atrial masses.

and was found to have multiple embolic lesions or infarcts on head computerized tomography (CT). Brain MRI revealed infarcts of the subcortical white matter, pons, and cerebellum. TEE demonstrated a $6 \mathrm{~mm}$ mobile, irregularly-shaped echodensity associated with the atrial surface of the mitral valve extending from the base of the interatrial septum to the intervalvular fibrosa (Fig. 2a). She was diagnosed with paroxysmal atrial fibrillation in the hospital, and warfarin (goal INR 2 - 3) was initiated; however repeat TEE 1 month later revealed a new $1.7 \times 1.1 \mathrm{~cm}$ pedunculated echodense mass within the RA arising from the posterior RA free wall and extending through the interatrial septum (Fig. 2b) (Supplementary Video 2a) (www. cardiologyres.org).

She underwent surgery under cardiopulmonary bypass. A right atrial incision perpendicular to the AV groove was made, exposing a $2 \times 1.5 \mathrm{~cm}$ pedunculated mass between the coronary sinus and septal leaflet of the tricuspid valve. It was excised with a small remnant of the posterior wall. A posterior left atrial incision was made and exposed a $0.5 \times 0.5 \mathrm{~cm}$ polypoid mass on the anterior leaflet of the mitral valve near the annulus, which was then excised. The interatrial septum was not involved. There were no postoperative events. Her etanercept was discontinued following surgery and her prednisone was reduced to $5 \mathrm{mg}$. Surgical pathology of her masses showed focal areas of fibrinoid necrosis surrounded by palisading histiocytes, chronic inflammatory cells, and giant cells which was diagnostic for rheumatoid nodules (Fig. 3). One month later she presented with a recurrent cerebrovascular accident despite a therapeutic INR; repeat TEE revealed new pedunculated masses in the RA and on the mitral and tricuspid valves, which did not have the

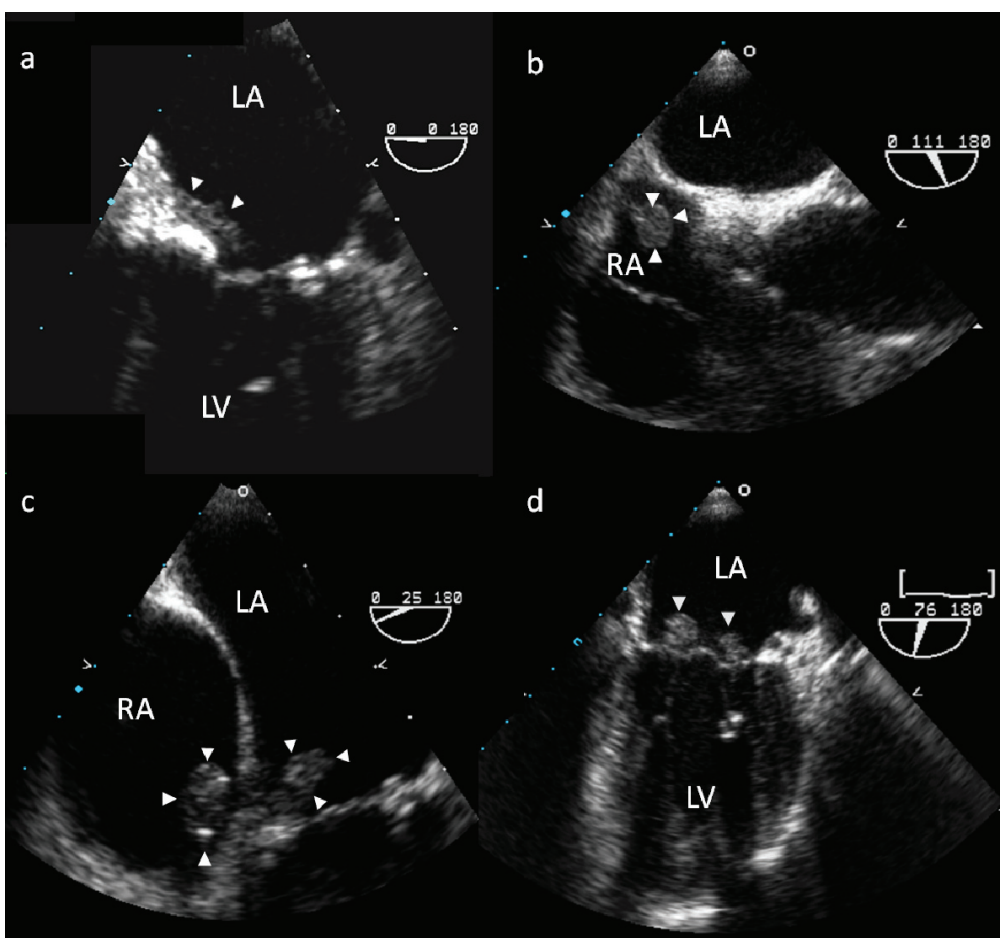

Figure 2. TEE of echodensities associated with the atrial surface of the mitral valve (a) and the posterior RA free wall (b). TEE 1 month following surgical resection shows new pedunculated masses in the RA and on the mitral and tricuspid valves (c, d). 


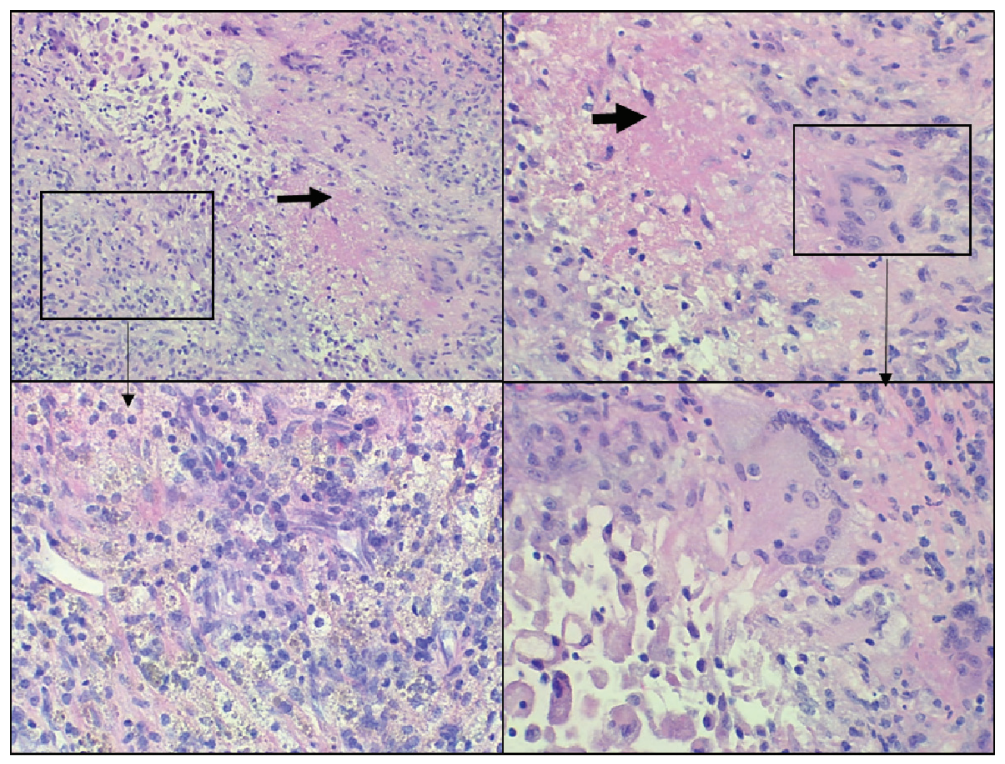

Figure 3. Surgical pathology images of right atrial mass. Lesional area showing necrosis (black arrow) containing eosinophilic debris surrounded by histiocytes, lymphocytes, plasma cells, and giant cells (box). These changes are compatible with rheumatoid nodule.

appearance of thrombus (Fig. 2c, d) (Supplementary Videos 2b, c) (www. cardiologyres.org). Her etanercept was restarted and repeat TEE 3 months later showed persistent $6 \times 8 \mathrm{~mm}$ mobile echodensities on the mitral valve; however, the previously seen masses in the RA and on the tricuspid valve had resolved. She was lost to follow-up and expired 4 years later from urosepsis.

\section{Case 3}

A 66-year-old woman presented with fevers and night sweats and was found to have new first degree atrioventricular block. Transthoracic echocardiogram (TTE) revealed an aortic root mass; TEE demonstrated a large complex mass invading the intraatrial septum, encasing the aortic root and extending into the right ventricular outflow tract (Fig. 4a). Blood cultures were negative. Inflammatory markers were consistent with Wegener's granulomatosis (+MPO ANCA). She developed high degree atrioventricular heart block and a dual chamber pacemaker was implanted. Cyclophosphamide and prednisone were started and serial TTEs showed progressive regression of the aortic mass. On repeat TEE the following year, the aortic mass was much smaller (Fig. 4b) (Supplementary Video 3) (www.cardiologyres.org). She underwent an aortic valve replacement with a $19 \mathrm{~mm}$ Carpentier-Edwards bioprosthetic valve soon afterwards due to development of severe aortic regurgitation; intense inflammatory-type tissue was noted below the non-coronary leaflet extending into the annulus and into the anterior leaflet of the mitral valve, essentially encompassing the fibrous trigone. Biopsy showed myxoid changes and chronic inflammation (Fig. 5). She continues to do well 6 years post surgery and most recent echocardiogram showed well functioning bioprosthetic aortic valve without recurrent mass.

\section{Discussion}

The therapeutic approach to the management of cardiac mass-

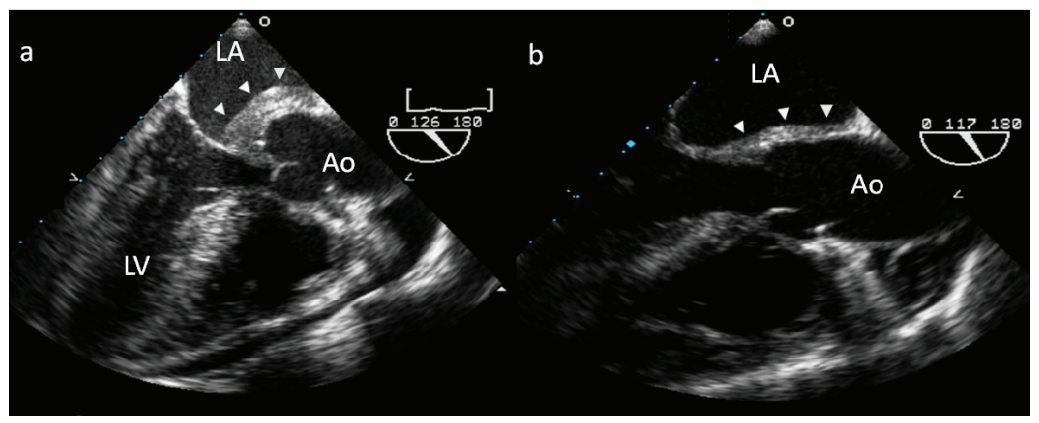

Figure 4. (a) TEE of a large complex mass invading the intaatrial septum and encasing the aortic root. Cyclophosphamide and prednisone were started and serial TTEs showed progressive regression of the aortic mass. (b) Follow-up TEE the following year shows near resolution. 


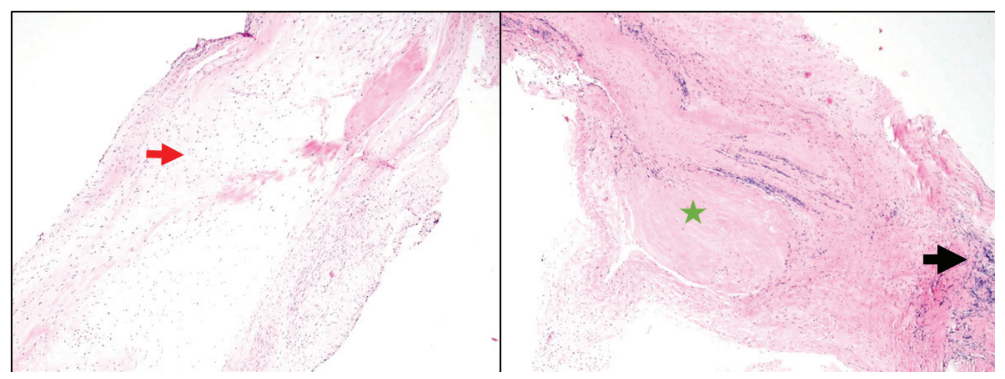

Figure 5. Surgical pathology images of valvular tissue: valvular tissue with myxoid changes (red arrow), focal chronic inflammation (black arrow), and fibrosis (green star). These changes could represent changes compatible with previously active vasculitis.

es depends on the nature of the mass. Surgical treatment for primary cardiac malignancies (predominantly sarcomas) and metastatic disease to the heart is often limited by the poor prognosis associated with these conditions; however, surgical resection is the treatment of choice for all benign cardiac tumors and in those malignant tumors which are potentially curable or can result in significant palliation. These tumors must, however, be differentiated from vegetation, thrombus and inflammatory pseudotumor (IP).

The term IP has been applied to a variety of inflammatory masses, including IMT. IMT is a rare lesion that usually presents as an intrapulmonary mass in children but may occur in adults. It is thought to arise from a benign tissue proliferative response and is composed of mesenchymal and polyclonal inflammatory cells. It commonly occurs in the lung, stomach, and orbit, but is rare in the heart [6]. Cardiac IMT is pathologically distinguishable from cardiac fibromas, myxomas, and sarcomas [16]; and may be biologically distinct from extracardiac IMT as evidenced by its usual lack of locally aggressive behavior [17]. Although there is no consensus on optimal therapy, there are four reported cases of cardiac IMT regressing either spontaneously [5] or following steroid treatment [4,
6]. However, the recurrence rate has been reported at 13 to $37 \%$ [18]. Furthermore, recent data suggests that IMT may be pathologically distinct from other forms of IP, and those associated with a chromosomal translocation in the ALK-1 tyrosine kinase have malignant potential [19].

Systemic inflammatory diseases are a family of autoimmune conditions that share common pathogenetic mechanisms and can be subclassified into vasculitides, connective tissue diseases, and miscellaneous disorders [20]. These conditions may involve multiple organ systems including the heart, and all components of the heart may be affected, including the valves, coronary arteries, conduction system, myocardium, endocardium or pericardium [21]. Furthermore, there have been several prior reports of IP developing in patients with disorders such as Wegener's granulomatosis [7, 8], sarcoidosis [9, $10]$, Behcet's disease [11, 12], and rheumatoid arthritis [1315]. These IPs may arise in the presence of a known systemic inflammatory disease or may be an isolated finding, and are likely pathologically distinct from IMT. Indeed, pathological examination of cardiac IP in patients with a known systemic inflammatory disease has been found to be histologically consistent with their systemic process [7-15].

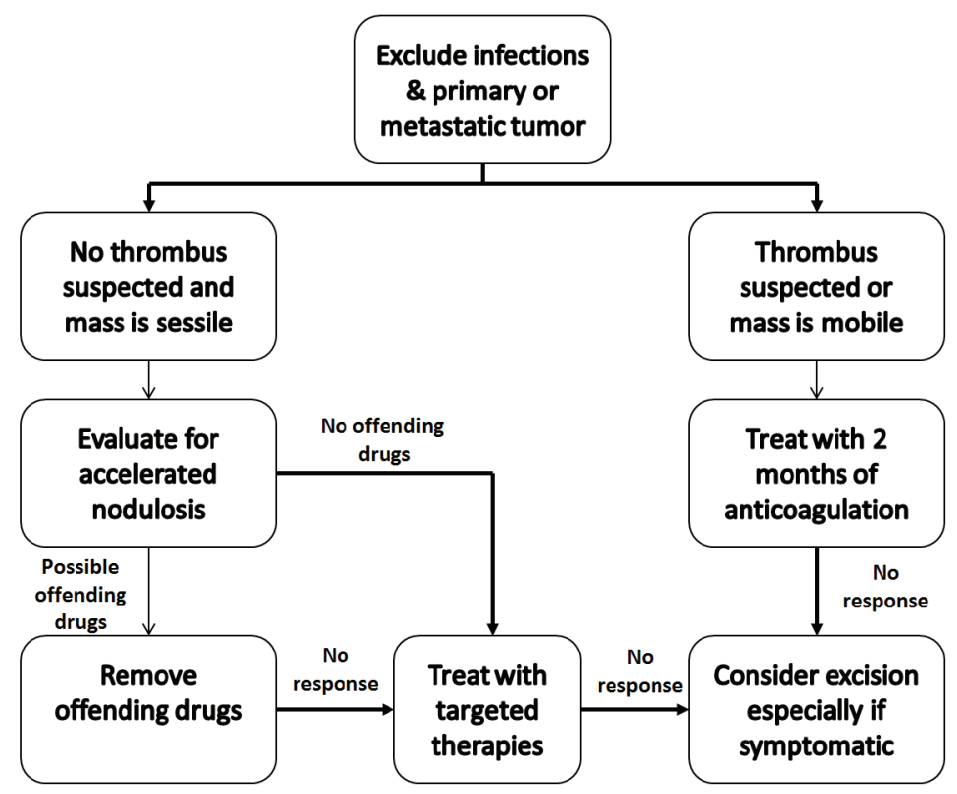

Figure 6. Proposed management strategy for suspected inflammatory cardiac masses. 
The optimal treatment of masses associated with systemic inflammatory disease is not well established; however, recurrence after surgical resection and regression after steroid therapy has been reported. For instance, subcutaneous rheumatoid nodules may recur after initial excision but often regress after conventional systemic treatment for rheumatoid arthritis or direct injection of corticosteroids [22]. Joffe et al [10] describe a patient who presented with palpitations and lightheadedness and was found to have right atrial and biventricular masses with biopsy consistent with sarcoidosis. High dose prednisone therapy resulted in significant reduction in size of the atrial mass and resolution of the ventricular mass. A similar therapeutic response was seen in the cases presented above. In case 1 there was near complete resolution of the IP after initiation of prednisone for rheumatoid arthritis, and in case 3 there was marked reduction in the size of the intracardiac masses with institution of prednisone and cyclophosphamide for Wegener's granulomatosis. Furthermore, in case 2, the rheumatoid nodules recurred after initial surgical resection, but regressed significantly after re-initiation of etanercept. It is, therefore, reasonable to postulate that in patients with IP associated with systemic inflammatory disease, treatment of the systemic condition may ameliorate cardiac manifestations as well, and a conservative non-surgical approach to the initial management of these lesions could be considered.

Careful evaluation of a patient's medications may identify another potential etiology of cardiac masses, as illustrated in case 1. Accelerated nodulosis, indistinguishable from rheumatoid nodules, typically occurs on hands or feet but may affect the heart or lung [23]. A number of drugs used to treat inflammatory diseases have been implicated including methotrexate, anti-tumor necrosis factor alpha drugs, and leflunomide [22]. While no clear consensus exists, management usually involves stopping the offending medication and/or adding an alternative anti-rheumatologic agent [24].

Before embarking on conservative management of a cardiac mass, the differential diagnosis needs to be carefully considered, including thrombus, infection, primary cardiac tumors, and metastatic lesions. Cardiac imaging can be useful to differentiate among these. Echocardiography is often the initial modality with which the mass is discovered. Echo contrast enhancement is a surrogate for vascularity; whereas thrombi have none, myxomas have partial, and malignant or highly vascular tumors have significant enhancement. Attachment of the mass at an area of wall motion abnormality make tumor more likely. Three-dimensional echo allows for volumetric assessment and analysis of various aspects of the mass [25]. On cardiac MRI, thrombi tend to be smaller, more heterogeneous and have a pattern of hyperintensity/isointensity compared to tumors. Malignant tumors tend to be larger and more often exhibit late gadolinium enhancement and contrast first-pass perfusion compared to benign tumors [26]. The role of catheter-based endomyocardial biopsy (EMB) for cardiac tumors is uncertain. Per the 2007 American College of Cardiology, American Heart Association, and European Society of Cardiology guidelines on EMB in management of cardiovascular disease, EMB is a class IIa indication if: 1) The diagnosis cannot be made with non-invasive modalities or non-cardiac biopsy; 2) Tissue diagnosis can be expected to alter the direction of therapy; 3) Chances of successful biopsy are reasonably high; 4) The procedure is performed by an experienced operator. Guidance with TEE or cardiac MRI is advised [27].

Thrombus should be suspected in patients with multiple or prolonged indwelling central lines, recent structural cardiac surgery, hypercoagulable states, and atrial fibrillation/flutter, especially those with subtherapeutic levels of anticoagulation [28]. Even large atrial thrombi may resolve following 6 - 8 weeks of anticoagulation with warfarin or low molecular weight heparin [29-32]. Therefore, in most cases it is reasonable to initiate a trial of oral anticoagulation as this can be curative. Furthermore, systemic anticoagulation should always be considered for mobile lesions to diminish risk of stroke, as inflammatory masses may have superimposed thrombus [33]. A case series of 22 patients with Behcet disease demonstrated that intracardiac thrombus can resolve with systemic immunosuppressive alone, presumably by reversing the hypercoagulable state responsible for the thrombus [34]. However, the sample size is too small to recommend universally withholding anticoagulation. Duration of anticoagulation is unclear as the inflammatory disease process may recur, putting the patient at risk for repeat thrombus. However, those with prior stroke or high CHA2DS2-VASc score would be candidates for long-term anticoagulation. Infection should be identified and treated. There are two reports of large right atrial masses consistent with tuberculomas on biopsy that completely resolved following 6 - 12 months of anti-tuberculous therapy $[35,36]$. In addition, IP has also been reported in association with other infections including Listeria monocytogenes [37, 38] and Mycobacterium avium-intracellulare [39]. Primary cardiac tumors are often suspected by their appearance on imaging studies and the primary source of metastatic disease to the heart is usually obvious on review of history, examination, and laboratory testing.

The potential pitfalls of a conservative approach to therapy include pulmonary or systemic embolization, stroke, and mechanical obstruction. The alternative to medical management is surgical excision via a traditional sternotomy with its attendant risks. Nonetheless, as illustrated by case 2, these masses may recur after initial surgical resection. A multi-modality and multi-disciplinary management strategy with input from cardiologists, cardiac surgeons, and imaging specialists is prudent. Based on our experience, we propose a treatment algorithm for suspected inflammatory cardiac masses (Fig. 6). First, infection should be ruled out. Thrombus and malignant and benign tumors can be differentiated with cardiac MRI and contrast echocardiography. Surgical excision is the treatment of choice for benign and some malignant tumors based on prognosis and symptoms. If thrombus is suspected, a trial of 2 months of therapeutic anticoagulation followed by repeat imaging is reasonable. If there is no response or the patient suffers stroke or obstructive symptoms, surgical excision is indicated. If neither tumor nor thrombus is present, evaluation for accelerated nodulosis should be performed and offending drugs removed. IMT would be the last remaining diagnosis for which immunosuppressive therapy may be trialed. If there is no response or the patient suffers stroke or obstructive symptoms, surgical excision is indicated. 


\section{Conclusions}

In summary, systemic inflammatory diseases are associated with a variety of cardiac manifestations including intracardiac masses. We present three such cases that were successfully treated with a conservative strategy and present an algorithmic approach for patients with suspected inflammatory cardiac masses. After excluding infection and primary or metastatic tumors, an initial trial of anticoagulation is reasonable for patients in whom thrombus is suspects, as even a large thrombus may readily respond. If there is no response to anticoagulation or thrombus is not suspected, therapy targeted at the underlying inflammatory disease is indicated and serial imaging studies should be obtained to assess the response. While the risk of systemic embolization, stroke, and cardiac obstructive syndromes exists with this approach, extirpation of intracardiac masses is by no means curative and recurrent lesions may form as seen in case 2. Ultimately, the approach to complex intracardiac masses must be individualized; the risks of surgery and the potential for tumor recurrence must be weighed against the risks of a conservative approach on a case-by-case basis.

\section{Acknowledgments}

We wish to thank Dr. Josenia Tan for providing pathology images and for Dr. Harold Lazar for reviewing the manuscript.

\section{Declaration}

None.

\section{Abbreviations}

IMT: inflammatory myofibroblastic tumor; MRI: magnetic resonance imaging; TEE: transesophageal echocardiogram; LA: left atrium; RA: right atrium; CT: computerized tomography; IP: inflammatory pseudotumor; EMB: endomyocardial biopsy

\section{References}

1. Reynen K. Frequency of primary tumors of the heart. Am J Cardiol. 1996;77(1):107.

2. Jain D, Maleszewski JJ, Halushka MK. Benign cardiac tumors and tumorlike conditions. Ann Diagn Pathol. 2010;14(3):215-230.

3. Palmer TE, Tresch DD, Bonchek LI. Spontaneous resolution of a large, cavernous hemangioma of the heart. Am J Cardiol. 1986;58(1):184-185.

4. Ferbend P, Abramson LP, Backer CL, Mavroudis C, Webb CL, Doll JA, Junewick JJ, et al. Cardiac plasma cell granulomas: response to oral steroid treatment. Pediatr Cardiol. 2004;25(4):406-410.
5. Pearson PJ, Smithson WA, Driscoll DJ, Banks PM, Ehman RL. Inoperable plasma cell granuloma of the heart: spontaneous decrease in size during an 11-month period. Mayo Clin Proc. 1988;63(10):1022-1025.

6. Tomiyama M, Nakatani S, Ishibashi-Ueda H, Yutani C, Yamagishi M. Inflammatory pseudotumor of the heart. Ann Intern Med. 2007;147(5):351-352.

7. Singh R, Rosen S. Tumor of the heart in a young woman; a rare manifestation of Wegener granulomatosis. Hum Pathol. 2012;43(2):289-292.

8. Herbst A, Padilla MT, Prasad AR, Morales MC, Copeland JG. Cardiac Wegener's granulomatosis masquerading as left atrial myxoma. Ann Thorac Surg. 2003;75(4):13211323.

9. Abrishami B, O'Connel C, Sharma O. Cardiac sarcoidosis with presentation of large left atrial mass. Curr Opin Pulm Med. 2004;10(5):397-400.

10. Joffe, II, Lampert C, Jacobs LE, Owen AN, Ioli A, Kotler $\mathrm{MN}$. Cardiac sarcoidosis masquerading as a metastatic tumor: the role of transthoracic and transesophageal echocardiography. J Am Soc Echocardiogr. 1995;8(6):933937.

11. Yao FJ, Liu D, Zhang Y, Yin S. Inflammatory pseudotumor of the right ventricle in a 35-year-old woman with Behcet's disease: a case report. Echocardiography. 2012;29(6):E134-136.

12. Leitao B, Machado F, Soares F, Souza H, Queiroz AC, Santiago MB. Myocardial inflammatory pseudotumor and multiple thromboses as a manifestation of Behcet disease. J Clin Rheumatol. 2009;15(5):252-253.

13. Abbas A, Byrd BF, 3rd. Right-sided heart failure due to right ventricular cavity obliteration by rheumatoid nodules. Am J Cardiol. 2000;86(6):711-712.

14. Suriani RJ, Lansman S, Konstadt S. Intracardiac rheumatoid nodule presenting as a left atrial mass. Am Heart J. 1994;127(2):463-465.

15. Webber MD, Selsky EJ, Roper PA. Identification of a mobile intracardiac rheumatoid nodule mimicking an atrial myxoma. J Am Soc Echocardiogr. 1995;8(6):961-964.

16. Obikane H, Ariizumi K, Yutani C, Mitsumata M. Inflammatory pseudotumor (inflammatory myofibroblastic tumor) of the mitral valve of the heart. Pathol Int. 2010;60(7):533-537.

17. Burke A, Li L, Kling E, Kutys R, Virmani R, Miettinen M. Cardiac inflammatory myofibroblastic tumor: a "benign" neoplasm that may result in syncope, myocardial infarction, and sudden death. Am J Surg Pathol. 2007;31(7):1115-1122.

18. Sunbul M, Cagac O, Birkan Y. A rare case of inflammatory pseudotumor with both involvement of lung and heart. Thorac Cardiovasc Surg. 2013;61(7):646-648.

19. Gleason BC, Hornick JL. Inflammatory myofibroblastic tumours: where are we now? J Clin Pathol. 2008;61(4):428-437.

20. Sherman BM, Haspel KL. Inflammatory diseases and the heart. Int Anesthesiol Clin. 2012;50(2):173-204.

21. Sitia S, Atzeni F, Sarzi-Puttini P, Di Bello V, Tomasoni L, Delfino L, Antonini-Canterin F, et al. Cardiovascular involvement in systemic autoimmune diseases. Autoimmun 
Rev. 2009;8(4):281-286.

22. Garcia-Patos V. Rheumatoid nodule. Semin Cutan Med Surg. 2007;26(2):100-107.

23. Rau R, Herborn G. Benefit and risk of methotrexate treatment in rheumatoid arthritis. Clin Exp Rheumatol. 2004;22(5 Suppl 35):S83-94.

24. Patatanian E, Thompson DF. A review of methotrexate-induced accelerated nodulosis. Pharmacotherapy. 2002;22(9):1157-1162.

25. Mankad R, Herrmann J. Cardiac tumors: echo assessment. Echo Res Pract. 2016;3(4):R65-R77.

26. Pazos-Lopez P, Pozo E, Siqueira ME, Garcia-Lunar I, Cham M, Jacobi A, Macaluso F, et al. Value of CMR for the differential diagnosis of cardiac masses. JACC Cardiovasc Imaging. 2014;7(9):896-905.

27. Cooper LT, Baughman KL, Feldman AM, Frustaci A, Jessup M, Kuhl U, Levine GN, et al. The role of endomyocardial biopsy in the management of cardiovascular disease: a scientific statement from the American Heart Association, the American College of Cardiology, and the European Society of Cardiology. Circulation. 2007;116(19):2216-2233.

28. Al-Ebrahim KE. Right atrial mass: the dilemma of diagnosis and when not to operate. Ann Thorac Surg. 2009;87(6):2005; author reply 2005.

29. Floris N, Gabriel L, Marchandise B. Successful anticoagulation for treatment of a giant left atrial thrombus. Acta Cardiol. 2010;65(6):713-715.

30. Senturk T, Kaderli AA, Yesilbursa D. Resolution of a giant atrial thrombus following anticoagulation therapy. Cardiovasc J Afr. 2008;19(1):28-30.

31. Marcu CB, Kramer C, Donohue TJ. Giant left atrial thrombus successfully treated with anticoagulation. Heart Lung Circ. 2007;16(1):55-56.

32. Chrzanowski L, Lipiec P, Kasprzak JD. Rapid resolution of large right atrial mass after anticoagulant therapy. Eur Heart J. 2006;27(17):2087.

33. Kang H, Baron M. Embolic complications of a mitral valve rheumatoid nodule. J Rheumatol. 2004;31(5):10011003.

34. Emmungil H, Yasar Bilge NS, Kucuksahin O, Kilic L, Okutucu S, Gucenmez S, Kalyoncu U, et al. A rare but serious manifestation of Behcet's disease: intracardiac thrombus in 22 patients. Clin Exp Rheumatol. 2014;32(4 Suppl 84):S87-92.

35. Rao VR, Jagannath K, Sunil PK, Madhusudana N. A rare disappearing right atrial mass. Interact Cardiovasc Thorac Surg. 2012;15(2):290-291.

36. Goyal NK, Saxena A, Chopra P. Complete resolution of a large intracardiac mass with medical treatment: an echocardiographic follow up. Heart. 2005;91(8):1046.

37. Adler A, Fimbres A, Marcinak J, Johnson A, Zheng X, Hasegawa S, Shulman ST. Inflammatory pseudotumor of the heart caused by Listeria monocytogenes infection. J Infect. 2009;58(2):161-163.

38. Uehara Yonekawa A, Iwasaka S, Nakamura H, Fukata M, Kadowaki M, Uchida Y, Odashiro K, et al. Infective endocarditis caused by Listeria monocytogenes forming a pseudotumor. Intern Med. 2014;53(9):1029-1032.

39. Wood C, Nickoloff BJ, Todes-Taylor NR. Pseudotumor resulting from atypical mycobacterial infection: a "histoid" variety of Mycobacterium avium-intracellulare complex infection. Am J Clin Pathol. 1985;83(4):524527. 